\title{
Identification of NPC1 as a novel SARS-CoV-2 intracellular target
}

Isabel Garcia-Dorival| ${ }^{1 *}$, Miguel Ángel Cuesta-Geijo ${ }^{1,2^{*}}$, Lucía Barrado-Gil1 ${ }^{1,2}$, Inmaculada Galindo ${ }^{1}$, Jesús Urquiza ${ }^{1}$, Ana del Puerto ${ }^{1}$, Carmen Gill ${ }^{2}$, Nuria Campillo², Ana Martínez ${ }^{2}$, Covadonga Alonso ${ }^{1+*}$.

${ }^{1}$ Dpt. Biotechnology, Instituto Nacional de Investigación y Tecnología Agraria y Alimentaria (INIA), Ctra. de la Coruña km 7.5, 28040 Madrid, Spain

${ }^{2}$ Centro de Investigaciones Biológicas Margarita Salas (CSIC), Ramiro de Maeztu 9, 28040 Madrid, Spain

* Both authors have equally contributed to this work

${ }^{* *}$ Corresponding author

\section{Abstract}

Niemann-Pick type C1 (NPC1) receptor is an endosomal membrane protein that regulates intracellular cholesterol trafficking, which is crucial in the Ebola virus (EBOV) cycle. The severe acute respiratory syndrome coronavirus 2 (SARSCoV-2) enters the cell by binding of the viral spike (S) protein to the ACE2 receptor. This requires S-protein processing either by the surface transmembrane serine protease TMPRSS2 for plasma membrane fusion or cathepsin $L$ for endosomal entry. Additional host factors are required for viral fusion at endosomes. Here, we report a novel interaction of the SARS-CoV-2 nucleoprotein $(\mathrm{N})$ with the cholesterol transporter NPC1. Moreover, small molecules interfering with NPC1 that inhibit EBOV entry, also inhibited human coronavirus. Our findings suggest an important role for NPC1 in SARS-CoV-2 infection, a common strategy shared with EBOV, and a potential therapeutic target to fight against COVID-19.

Keywords: SARS-CoV-2, N protein, NPC1, target, antivirals. 
Introduction

To date, the COVID-19 pandemic has caused over one million of deaths and affected over 73-millions of people around the world (1). COVID-19 is caused by the emerging and pathogenic severe acute respiratory syndrome coronavirus 2 (SARS-CoV-2) first reported in the city of Wuhan (China) as a rare pneumonia $(2,3)$ that rapidly spread worldwide. A better understanding of the biology of SARS-CoV-2 is vital in order to develop effective therapeutics. A key step of the biology of SARS-CoV-2 is the cell entry mechanism. Viral entry involves the activation of its trimeric spike glycoprotein by TMPRSS2 protease upon interaction with the angiotensin-converting enzyme 2 (ACE2) receptor at the plasma membrane to mediate fusion (4-8). Also, the virus can enter via endosomes under cathepsin L processing, similar to SARS-CoV-1 (7, 9). In fact, the inhibition of both alternative entries is necessary for full inhibition of SARSCoV-2 entry $(10,11)$. Other cellular proteases facilitate SARS-CoV-2 cell tropism, like furin, possibly at a post-binding step $(12,13)$.

Considering that SARS-CoV-2 may enter the cell by endocytosis, it would be partially acid $\mathrm{pH}$ dependent and should exit endosomes by fusion to start replication (13). SARS-CoV-2 replication occurs in the cytoplasm at double membrane vesicles of possible origin in the endoplasmic reticulum, which provide support to the viral RNA replication and transcription complex (14-16).

SARS-CoV-2 endosomal pH-dependent masking through endosomal cleavage was recently described (8). Similarly, endosomal processing of the Ebola virus (EBOV) glycoprotein trimer enhances infectivity and evidence a conformational masking of the receptor binding site of this protein $(17,18)$. A similar process has been described for the human immunodeficiency virus (HIV) envelope trimer (19). Therefore, the similarities found between these viruses were the start point of this work. EBOV enters the cell using the endocytic pathway, and its entry is mediated by the viral glycoprotein (GP), which decorates the viral surface organized in trimeric spikes (20). The GP is then processed by endosomal cathepsins to remove its heavily glycosylated $\mathrm{C}$-terminal residues and the glycan cap. This removal produces the cleaved form of the $\mathrm{N}$-terminal receptor binding subunit GP1 ( $\mathrm{GP} \mathrm{CL}_{\mathrm{L}}$ that is required to mediate fusion at the endosomal membrane. Two simultaneous publications described first that the endosomal receptor called 
64 NPC1 is an intracellular host receptor for $\operatorname{EBOV}(21,22)$. Since then, the 65 relevance of NPC1 on viral infections have been shown for different viruses 66 including HIV (23), Hepatitis C (HCV) $(24,25)$, Chikungunya virus (CHIKV) (26) 67 and several Flaviviruses such as Dengue (DENV) $(27,28)$ and ZIKA virus (29) 68 among others $(26,30)$.

69

70 Given SARS-CoV-2 can be internalized via clathrin- and non-clathrin-mediated 71 endocytosis; some researchers hypothesized a role for NPC1 in SARS-CoV-2 72 infection that remains to be determined (31-34). Due to the increasing relevance 73 of this molecule, we designed our study to investigate the potential binding of 74 SARS-CoV-2 proteins to NPC1, according to the existing evidence of SARS75 CoV-2 endosomal passage (35). 


\section{Materials and Methods}

\section{Cell culture and viruses}

Human embryonic kidney cells 293T/17 (HEK 293T; ATCC-CRL-11268) were cultured in Dulbecco modified Eagle medium (DMEM) at $37 \stackrel{\circ}{\circ}$ and $5 \% \mathrm{CO}_{2}$ atmosphere, supplemented with $100 \mathrm{IU} / \mathrm{ml}$ penicillin, $100 \mu \mathrm{g} / \mathrm{ml}$ streptomycin, $1 \mathrm{X}$ GlutaMAX (Thermo Fisher) and 10\% heat-inactivated fetal bovine serum (FBS). Huh-7 Lunet C3 cells, a gift from T. Pietschman (Twincore, Germany), were cultured at $37^{\circ} \mathrm{C}$ in Dulbecco's modified Eagle's medium (DMEM) supplemented with $100 \mathrm{IU} / \mathrm{ml}$ penicillin, $100 \mu \mathrm{g} / \mathrm{ml}$ streptomycin, 10mM HEPES, 1X NEAA and $10 \%$ of heat-inactivated fetal bovine serum (FBS).

For virus infections, we used common cold coronavirus 229E, which expresses the green fluorescent protein (GFP) gene (229E-GFP) (36). This recombinant virus was kindly given by V. Thiel, at the University of Bern, in Switzerland. The infection experiments were conducted at $33^{\circ} \mathrm{C}$ and $5 \% \mathrm{CO}_{2}$.

Design and construction of plasmid that express the SARS-CoV-2 N tag to EGFP.

The methodology used in this part of the study was previously used in GarciaDorival et al., 2016 (37). To generate the SARS-CoV-2 N with N-terminal EGFP tag (EGFP-N), a codon optimized cDNA sequence for the ORF of SARS-CoV-2 N (NCBI reference sequence number: NC_045512) was cloned into the pEGFPC1 (by GeneArt-Thermo Fisher Scientific). Once cloned, the sequence of the plasmid EGFP-N was confirmed by sequencing (Gene Art-Thermo Fisher Scientific).

\section{Expression of tagged-N protein and EGFP in HEK 293T cells.}

104 To transfect HEK 293T cells, four $60 \mathrm{~mm}$ dishes were seeded with $2.5 \times 10^{6}$ cells 105 each 24 hours prior to transfection in DMEM complete medium described above. 106 Then, a transfection of EGFP or EGFP-N was done using Lipofectamine 2000

107 (Thermo Fisher Scientific), following the instructions of the manufacturer. Twenty108 four hours post transfection the cells were harvested, lysed and 109 immunoprecipitated using a GFP-Trap kit (Chromotek). 


\section{Immunoprecipitations (IP)}

112 EGFP-N and EGFP immunoprecipitations (IP) were done using a GFP-Trap ${ }^{\circledR} \_A$

113 (Chromotek). To do the IPs, the cell pellet was resuspended in $200 \mu$ l of lysis

114 buffer (10mM Tris/Cl pH 7.5; $150 \mathrm{mM} \mathrm{NaCl} ; 0.5 \mathrm{mM}$ EDTA; 0.5\%NP40) and then

115 incubated for 30 minutes on ice. The lysate was then clarified by centrifugation at

$11614000 \times g$ and diluted five-fold with dilution buffer $(10 \mathrm{mM} \mathrm{Tris} / \mathrm{Cl} \mathrm{pH} 7.5 ; 150 \mathrm{mM}$

$117 \mathrm{NaCl}$; 0.5mM EDTA). The GFP-Trap agarose beads were equilibrated with ice-

118 cold dilution buffer and then incubated with diluted cell lysate overnight at $4^{\circ} \mathrm{C}$ on

119 a rotator, followed by centrifugation at $2700 \times g$ for 2 minutes. The bead pellet

120 was wash two times with wash buffer $(10 \mathrm{mM}$ Tris $/ \mathrm{Cl}$ pH $7.5 ; 150 \mathrm{mM} \mathrm{NaCl} ; 0.5 \mathrm{mM}$

121 EDTA). After removal of wash buffer, the beads were resuspended in $100 \mu \mathrm{l}$ of

122 Sample Buffer, Laemmli 2 X Concentrate (Sigma Aldrich) and boiled at $95^{\circ}$ for ten

123 minutes to elute the bound proteins. Buffers used for Immunoprecipitations were

124 all supplemented with Halt ${ }^{\mathrm{TM}}$ Protease Inhibitor Cocktail EDTA-Free (Thermo

125 Fisher Scientific).

\section{Co-Immunoprecipitation (Co-IP)}

128 Similar to what was described in Garcia-Dorival et al, 2016 (37), Co-IP for NPC1 129 was performed using $50 \mu \mathrm{l}$ of the Immobilized Recombinant Protein G Resin 130 (Generon) and specific antibodies against NPC1 (Abcam, ab108921). The cell 131 pellets were incubated for 30 minutes on ice with $200 \mu$ l lysis buffer. The lysate 132 was then clarified by centrifugation and diluted five-fold with dilution buffer prior 133 to adding $2 \mu \mathrm{g}$ of the primary antibody and then incubated at $4^{\circ} \mathrm{C}$ on a rotator for 134 two hours. The protein $\mathrm{G}$ resin (Generon) were equilibrated with ice-cold dilution 135 buffer and then incubated at $4^{\circ} \mathrm{C}$ on a rotator with diluted cell lysate containing 136 the antibody overnight at $4^{\circ} \mathrm{C}$ on a rotator, followed by centrifugation at $2500 \mathrm{xg}$ 137 for 2 minutes to remove non-bounds fractions. The wash and elution steps were 138 performed as describe previously in GFP co-immunoprecipitation.

\section{Western blot analysis}

141 To do confirm the expression of GFP and GFP-N proteins, an SDS-PAGE and a 142 western blot (WB) was done. For the SDS-PAGE, a Mini-PROTEAN TGX Gels were used (Bio-Rad 4561096), then, the gels were transferred to PVDF 
144 membranes using the Trans-Blot Turbo Transfect Pack (Bio-Rad 1704159) and 145 the Trans-Blot Turbo system (Bio-Rad). Following this, the transferred 146 membranes were then blocked in 10\% skimmed milk powder dissolved in TBS$1470.1 \%$ Tween (TBS-T) $(50 \mathrm{mM}$ Tris- $\mathrm{HCl}(\mathrm{pH} 8.3), 150 \mathrm{mM} \mathrm{NaCl}$ and $0.5 \%(\mathrm{v} / \mathrm{v})$ 148 Tween-20) buffer for one hour at room temperature. Primary antibody was diluted 149 1:1000 in blocking buffer and then incubated at $4^{\circ} \mathrm{C}$ overnight. After three 150 washes, blots were incubated with appropriate HRP secondary antibody diluted 151 in blocking buffer at a 1:5000 for 1 hour at room temperature. Blots then were 152 developed using enhanced chemiluminescence reagent (Bio-Rad) and detected 153 with ChemiDoc ${ }^{\mathrm{TM}}$ XRS Gel Imaging System using Image Lab $^{\mathrm{TM}}$ software (Bio$154 \mathrm{Rad})$.

\section{Production of SARS-CoV-2 N protein in the baculovirus system}

157 The sequence of the N protein published in the NCBI database was selected 158 (GenBank accession number: 43740575 / NCBI reference sequence number: 159 NC_045512). The codon usage of the $\mathrm{N}$ encoding gene was optimized for its 160 expression in insect cells (OptimumGene ${ }^{\mathrm{TM}}$-Codon Optimization algorithm) and 161 the coding sequence for this protein was synthesized by the company GenScript.

162 The donor plasmid pFastBac1 containing an expression cassette expressing the 163 recombinant protein under the control of the polyhedrin promoter was obtained. 164 The Bacmid for the generation of the baculovirus was prepared in E. Coli DH10Bac bacterial cells containing the mini-Tn-7-replicon. Bacmids were transfected in the regulatory Sf9 cells and a viral clone selection was made by two rounds of plaque cloning to obtain the working virus stock. The baculovirus genome region was sequenced to determine the integrity of the $\mathrm{N}$ gene in the recombinant baculovirus named $\mathrm{rBacN}$.

\section{SARS-CoV-2 $\mathrm{N}$ protein production in pupae}

172 The production of SARS-CoV-2 N protein in insect pupae (Tricoplusia ni; T. ni) 173 was performed as previously described (38). Briefly, pupae were allocated in the 174 inoculation robot that dispensed a maximum of $5 \mu \mathrm{l}$ with the baculovirus titers 175 protein in 5 days pupae incubation time in constant temperature and humidity 176 chambers. After that period, pupae were collected and stored frozen, before 177 downstream processing. T.ni pupae containing the recombinant protein were 
178 homogenized in extraction buffer. Then, subsequent steps of clarification,

179 diafiltration and His-tag purification were carried, out in order to obtain purified

180 SARS-CoV-2 N protein. Protein concentration, yield and level of purity were 181 determined by SDS-PAGE analysis using 4-20\% or $12 \%$ Mini-Protean TGX 182 precast gels from Bio-Rad. Gels were stained with QC Colloidal stain (3 ng 183 sensitivity) in the case of concentration and yield evaluation and with SYPRO 184 Ruby (1 ng sensitivity) in the case of level purity analysis, both from Bio-Rad. 185 Recombinant SARS-CoV-2 N protein produced in pupae was measured by band 186 densitometry with the ChemiDoc ${ }^{\mathrm{TM}}$ XRS Gel Imaging System using Image Lab ${ }^{\mathrm{TM}}$ 187 software (Bio-Rad). A BSA standard curve was used for quantification.

\section{ELISA assays}

190 High-binding 96-well ELISA plates (Nunc) were coated with $0.5 \mu \mathrm{g} /$ well of purified SARS-CoV-2 N protein in carbonate/bicarbonate buffer $0.05 \mathrm{M} \mathrm{pH} 9.6$ and allowed to bind over night at $4^{\circ} \mathrm{C}$. Then, endogenous human NPC1 and HSP90 were purified using immobilized Recombinant Protein G Resin (Generon) and 4 $\mu \mathrm{g}$ of specific antibodies against NPC1 (Abcam, ab108921) or HSP90 (Enzo Life Sciences, ADI-SPA-835) respectively. All steps were performed as described in Co-IP assays. Serial dilutions of these endogenous NPC1 and HSP90 were added to the plate and capture was allowed to proceed for 1 hour at $37^{\circ} \mathrm{C}$. After that, plates were washed with PBST (PBS 0.1\%Tween20) and the binding of NPC1 to SARS-CoV-2 N protein was detected with a rabbit anti-NPC1 antibody (1:2000), revealed with an anti-rabbit-horseradish peroxidase (HRP) (1:2000) using a colorimetric substrate (OPD) and finally, quantified by absorbance at 492 $\mathrm{nm}$ in the EnSight multimode plate reader of PerkinElmer. Effect of the compounds on the binding was performed pre-incubating $50 \mu \mathrm{M}$ and $100 \mu \mathrm{M}$ of each compound with NPC1 $1 \mathrm{~h}$ a $37^{\circ} \mathrm{C}$ before adding it to the plate.

\section{Compounds studied}

All the compounds tested in this work have a purity $\geq 95 \%$ by HPLC. SC compounds were synthesized at Centro de Investigaciones Biológicas (CIBCSIC) following described procedures. All these molecules were included in the MBC chemical library and some of them were previously characterized as 
$212 \operatorname{GP}(39,40)$. The compounds tested in this study are shown in Figure 2 and were

213 resuspended in DMSO at $50 \mathrm{mM}$. Sulfides SC198 and SC073, and carbazole

214 SC816, were used at working concentrations of 5,50 and $50 \mu \mathrm{M}$;

215 benzothiazepines SC397, SC593, SC567, at working concentrations of $75 \mu \mathrm{M}$

216 and SC338 at $100 \mu \mathrm{M}$ respectively. The first three compounds were shown

217 previously to be active against EBOV while the others were inactive (40).

218 Compounds MBX2254 and MBX2270 were used as gold standards as they have

219 been reported to inhibit EBOV-GP/NPC1 interaction with high selectivity (41).

220 These compounds were purchased from MolPort and used at concentrations of

$22175 \mu \mathrm{M}$ and $25 \mu \mathrm{M}$, respectively. Class II cationic amphiphilic compound U18666A

222 is a drug that blocks cholesterol flux out of lysosomes and also inhibits Ebola

223 virus infections. It was acquired from Sigma-Aldrich and used at $10 \mu \mathrm{M}$ (42).

224 Imipramine, a hydrophobic amine and FDA-approved antidepressant drug was

225 acquired from Sigma-Aldrich and used at $25 \mu \mathrm{M}(26,43)$.

\section{Cytotoxicity assays}

228 Huh-7 cells were seeded in 96-well plates and incubated with DMEM containing 229 each compound at concentrations ranging from 0 to $100 \mu \mathrm{M}$. After 24 hours, cell

230 viability was measured by Cell Titer 96 AQueous Non-Radioactive Cell 231 Proliferation Assay (Promega) following the manufacturer's instructions.

232 Absorbance was measured at $490 \mathrm{~nm}$ using an ELISA plate reader.

233 Cell viability was reported as the percentage of absorbance in treated cells 234 relative to DMSO- treated cells (Figure S3). The 50\% cytotoxic concentration $235\left(\mathrm{CC}_{50}\right)$ was calculated and non-toxic working concentrations (over $80 \%$ cell 236 viability) used to test the activities of these compounds on CoV infection.

237 The values of the half maximal inhibitory concentration $\left(\mathrm{IC}_{50}\right)$ inhibition of the 238 infection presented on Figure 3 table correspond to the mean of 3 independent 239 experiments. The $\mathrm{IC}_{50}$ S values and dose-response curves were estimated using 240 GraphPad Prism v6.0 with a 99\% confidence interval.

\section{Flow cytometry analysis}

243 Detection of CoV infected cells was performed by flow cytometry. Huh-7 cells 244 were pre-treated with compounds at the indicated concentrations in growth 245 medium for $1 \mathrm{~h}$ at $33 \stackrel{\circ}{\circ}$, followed by infection with $229 \mathrm{E}-\mathrm{GFP}$ at a multiplicity of 
246 infection (MOI) of $1 \mathrm{pfu} / \mathrm{cell}$ for $24 \mathrm{~h}$. Cells were washed twice with growth medium

247 after 90 min of adsorption at $33^{\circ} \mathrm{C}$, and incubated with DMEM $10 \% 24 \mathrm{~h}$. Cells

248 were then harvested with PBS-EDTA 5mM, and diluted in PBS. Detection of

249 229E-GFP infected cells was performed by analyzing GFP expression. In order

250 to determine the percentage of infected cells per condition, 8,000 cells/time point

251 were scored using FACS Canto II flow cytometer (BD Sciences) and analyzed

252 using the FlowJo software. Untreated control infected cultures yielded $75-90 \%$

253 of infected cells from the total cells examined. Infected cell percentages obtained

254 after drug treatments were normalized to DMSO values.

256 Statistical analysis

257 The experimental data was analyzed by one-way ANOVA by Graph Pad Prism 6 258 software. For multiple comparisons, Bonferroni's correction was applied. Values

259 were expressed in graph bars as mean \pm SD of at least three independent 260 experiments unless otherwise noted. A $p$ value $<0.05$ was considered as 261 statistically significant. 


\section{Results}

\section{Interaction of SARS-CoV-2 N protein with NPC1}

To investigate the interaction of SARS-CoV-2 Nucleoprotein (N) with NPC1, N protein was expressed as an EGFP-fusion protein in HEK 293T cells. Then, proteins were extracted from lysed cells and assayed for immunoprecipitation (IP) using a high affinity EGFP immunoprecipitation kit (GFP-Trap). Finally, protein expression/interaction was confirmed by fluorescence and western blot analysis (Figure 1A and 1C). This pipeline (Figure 1B) has been used to detect protein

272 interacting partners for other RNA virus such as EBOV (37, 44). HEK 293T cells were selected due to their high efficiency of transfection, being the cell line of choice for protein-protein interaction studies of several viruses including SARSCoV-2 (45).

Protein expression of EGFP-N and EGFP alone was confirmed using western blot analysis and fluorescence (Figure 1A and 1C). The efficiency of transfection for both plasmids was approximately $80 \%$ (Figure 1A). EGFP-N and EGFP were then immunoprecipitated using an EGFP-Trap. After immunoprecipitation, both input (cell lysate) and bound (or elution) samples were analysed by western blot. Proteins corresponding to the molecular weight of EGFP-N (70 kDa) and the EGFP empty (27 kDa) were detected using an anti-EGFP antibody (Figure 1C). NPC1, as an endogenous protein, was also detected in both input samples (EGFP-N and EGFP); but only in the bound fraction of EGFP-N sample (Figure 1C). This experiment was repeated three times to ensure reproducibility (Supplementary figure S1A).

To further validate a specific interaction between EGFP-N and NPC1, two cellular proteins were selected as negative controls. In this case, HSP90 chaperone and endosomal protein EEA1 were used as controls given the abundance of these proteins in cells (Figure 1C).

\section{Validation of SARS-CoV-2 N interaction with NPC1}

293 Co-immunoprecipitations against NPC1 (or reverse pull down) were performed

294 to confirm and further validate the interaction between SARS-CoV-2 $\mathrm{N}$ and NPC1. SARS-CoV-2 N was overexpressed in HEK 293T cells and then cellular 
298 obtained from the co-immunoprecipitations were then analysed by western blot, which confirmed the presence of SARS-CoV-2 N (Figure 1D). As a result of this biology.

A
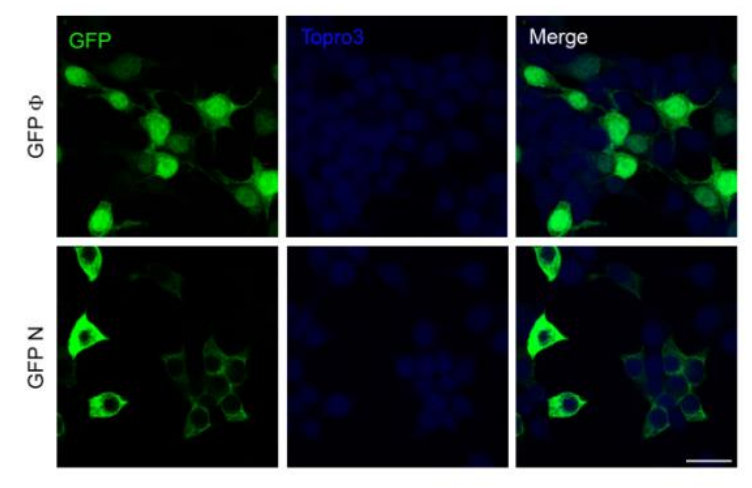

C

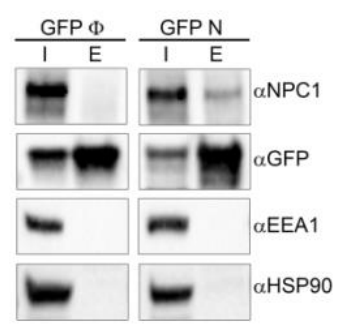

D

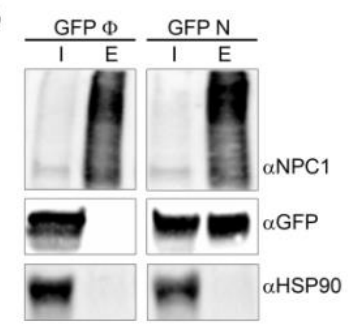

B
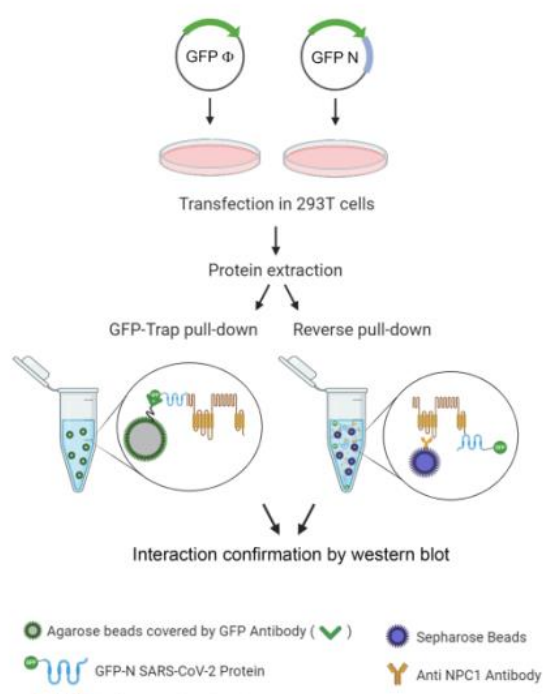
$\checkmark$ Anti NPC1 Antibody
302

303

304

305

306

307

308

309

310

311

312

313

314

\section{5}

316

317

318

319

320

Figure 1. Immunoprecipitation analysis of SARS-CoV 2 N protein with endogenous NPC1.

A. Expression of SARS-CoV-2 Nucleoprotein. Immunofluorescence of HEK 293T cells transiently expressing EGFP at the upper panel and EGFP-N protein at the lower panel showing different distribution as expected. GFP, Topro3 and Merge are indicated in upper panels in different colours. The scale bar indicates $25 \mu \mathrm{m}$. B. Schematic representation of the methodology used in this study for immunoprecipitation. C, D. Detection of SARS-CoV-2 N fussed to GFP, GFP control and cellular proteins analysed in the immunoprecipitation assay by western blot. C. Endogenous NPC1, EEA1, HSP90; and transfected EGFP$\mathrm{N}$ and EGFP control were detected at the expected molecular weights. D. Endogenous NPC1, HSP90 and transfected EGFP-N and EGFP control were detected at the expected molecular weights from samples collected from the co-immunoprecipitations (reverse pulldown). Molecular weighs: NPC1 175kD, EEA1 110kD, HSP90 75kD, EGFP-N 70kD, EGFP 27kD.

\section{Functional assays}

For an orthogonal characterization of the interaction, we used NPC1 inhibitor drugs to inhibit human coronavirus (HCoV) infection. To do this, Huh-7 cells were treated with the inhibitor compounds for an hour at different concentrations and then, infected with $\mathrm{HCoV} 229 \mathrm{E}-G F P$ recombinant virus at a $\mathrm{MOI}$ of $1 \mathrm{pfu} / \mathrm{ml}$. Cells were then analysed at $16 \mathrm{hpi}$. 


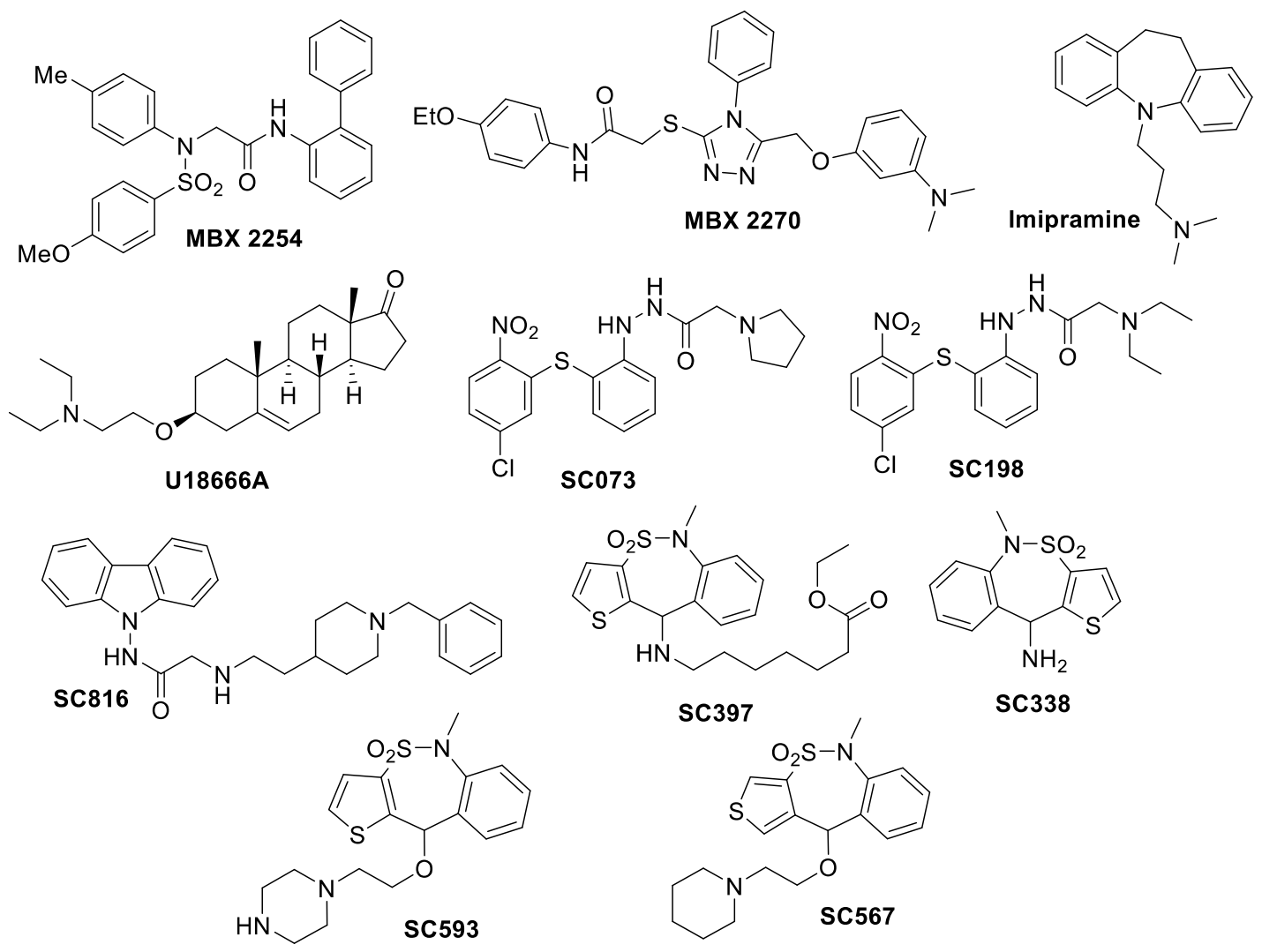

Figure 2. Chemical structure of small molecules used in this study.

Inhibitors MBX2254 and MBX2270 (Figure 2) were selected as they target NPC1 with high selectivity and both have been described to inhibit HIV-pseudotypedEBOV-GP binding to NPC1 (41). MBX2254 and MBX2270 were used at 75 and $25 \mu \mathrm{M}$, respectively. We also used imipramine, a Food and Drug Administration (FDA)-approved drug, that inhibits EBOV and other viruses due to its ability to induce a phenotype similar to NPC1 deficiency (46).

331 Finally, we assayed a set of compounds initially selected by virtual screening of

332 the MBC chemical library in the EBOV-GP/NPC1 interaction (40). These compounds were previously found to inhibit infection with EBOV pseudotyped retrovirus and some of them - sulfides and carbazoles - were able to disturb the NPC1-GP interaction in an ELISA assay. Compounds were classified in three chemical classes, sulfides SC198 and SC073, and carbazole SC816 used at 5, 50 and $50 \mu \mathrm{M}$ respectively; and benzothiazepines SC397, SC593, SC567 (Figure 2), that were used at $75 \mu \mathrm{M}$, or $100 \mu \mathrm{M}$ of SC338. Noteworthy, sulfides and carbazoles were found to potentially act through inhibition of NPC1-GP 340 interaction, while benzothiazepines do not affect this interaction (40). Based on 
341 these previous results, the three classes were included in this study for

342 comparative purposes. As a reference, we used U18666A compound (10 $\mu \mathrm{M})$,

343 known to inhibit cholesterol transport function of NPC1 and the infectious entry of

344 several viruses including EBOV and $\operatorname{ASFV}(23,26,42)$.

345 We detected that MBX2270 derivative potently inhibited HCoV infection (50\% 346 inhibitory concentration $\mathrm{IC}_{50}=3.26 \mu \mathrm{M}$, selectivity index 28.36; Figure 3). In

347 general, our results yielded significant inhibition $>99 \%$ of $\mathrm{HCoV}$ infection with the

348 U18666A compound and imipramine treatment and with sulfides from the library 349 compounds (Figure $3 \mathrm{~A}$ ). Others yielded over $80 \%$ of infectivity inhibition (except 350 for SC397 and SC338; Figure 3B). IC 50 was $<1 \mu \mathrm{M}$ in several sulfide compounds 351 (SC073 IC $50=0.53 \mu \mathrm{M}$, Index 151.47), U18666A (IC $50.1 \mu \mathrm{M}$, Index 362.34) and 352 imipramine (FDA085; IC $\mathrm{C}_{50}=0.75 \mu \mathrm{M}$, Index 72.3). Full information of 353 dose/response curves for these chemicals are included in Supplementary Fig. 354 S4.

355

356

357

358

359

360

361

362

363

364

365

366

367
A

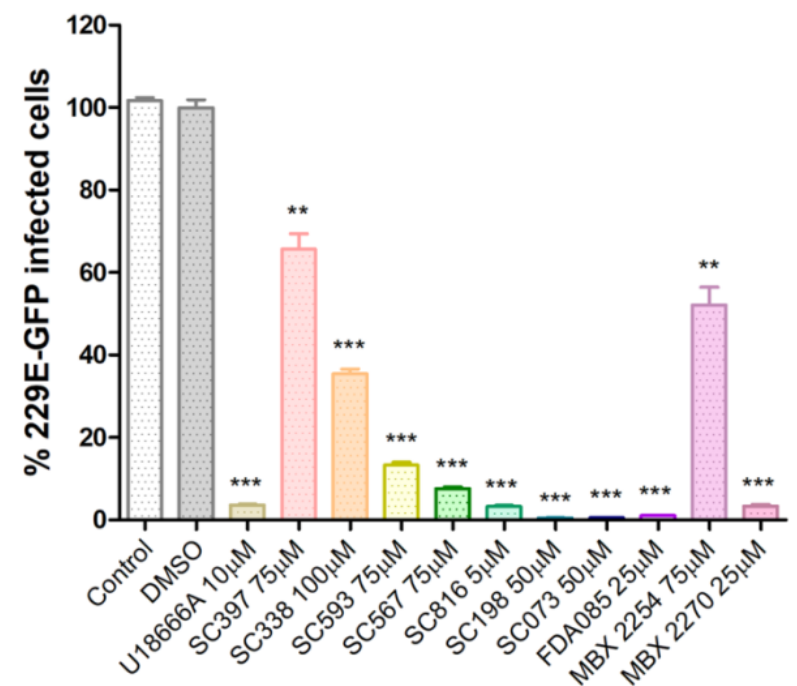

B

\begin{tabular}{r|ccc} 
& CC50 & IC50 & CC50/IC50 \\
\hline U18666A & 36,56 & 0,1009 & 362,34 \\
SC397 & $>100$ & & \\
SC338 & $>100$ & 35,92 & $>2,78$ \\
SC593 & $>100$ & $\sim 25.80$ & $>3,88$ \\
SC567 & $>100$ & 27,13 & $>3,69$ \\
SC816 & 8,591 & 0,9552 & 8,99 \\
SC198 & 112,6 & 2,611 & 43,12 \\
SC073 & 81,63 & 0,5389 & 151,47 \\
FDA085 & 54,37 & 0,752 & 72,30 \\
MBX 2270 & 92,53 & 3,263 & 28,36
\end{tabular}

Figure 3. Activity of small-molecule inhibitors of NPC1 and related compounds against HCoV. A. Infectivity percentages of HCoV 229E-GFP in Huh-7 cells at 24 hpi. Y axis depicts GFP fluorescence intensity in controls and cells pretreated $1 \mathrm{~h}$ before infection with selected compounds at the concentrations above described $\left({ }^{* *} p<0.001 ;{ }^{* *} p<0.0001\right)$. B. $I_{50}$ values $(\mu \mathrm{M})$ were determined for these compounds. For determination of $\mathrm{CC}_{50}$ values, cells were treated with compound alone, and values $(\mu \mathrm{M})$ were determined from linear portions of the dose-response curves shown in Supplementary Figure S4. SI, selectivity index $\left(\mathrm{CC}_{50} / \mathrm{IC}_{50}\right)$.

In addition to these functional experiments, we also tested the ability of these compounds to disrupt the NPC1/SARS-CoV-2 N protein interaction in an ELISA assay, as described in Materials and Methods. First, we tested increasing concentrations of NPC1 and control protein HSP90 in plates coated with SARS- 
368 CoV-2 N protein. We detected a positive reaction with increasing concentrations 369 of NPC1, while negative control HSP90 remained unaltered (Figure 4A). Then, 370 we analysed the inhibition of NPC1/SARS-CoV-2 N binding with a sample of the 371 compounds previously described in this study. We obtained a significant inhibition 372 of this specific binding in those samples tested with one inhibitor compound from 373 each class $100 \mu \mathrm{M}$ SC073 and 50 or $100 \mu \mathrm{M}$ of MBX2270 (Figure 4B).

A

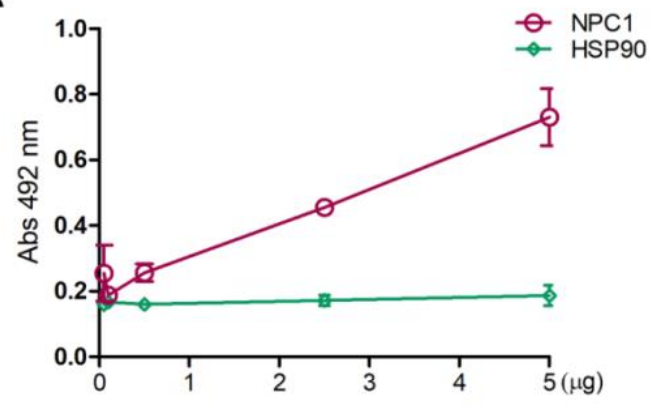

B

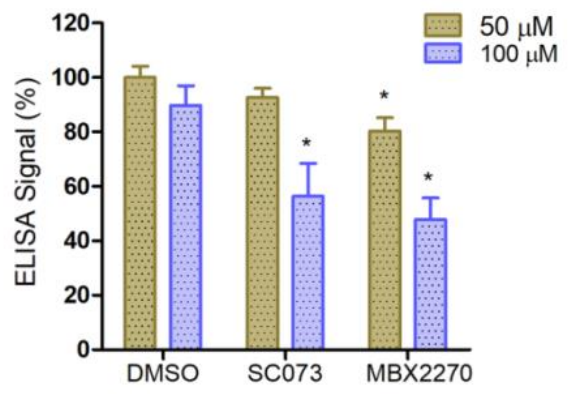

374

375

376

Figure 4: Inhibition of NPC1 binding to N protein by chemicals in an ELISA assay. A. Binding of increasing concentrations of NPC1 or HSP90 to N protein. HSP90 was used as a negative control. Concentrations analyzed were $5,2.5,0.5,0.1$ or $0.05 \mu \mathrm{g}$. B. DMSO or $50 / 100 \mu \mathrm{M}$ chemical compounds were incubated with $5 \mu \mathrm{g}$ of purified endogenous NPC1 before being added to ELISA plates previously coated with purified $\mathrm{N}$ protein $(0.5 \mu \mathrm{g} /$ well). Then, the binding of purified NPC1 protein to viral N protein was determined with an anti-NPC1 antibody revealed with an anti-rabbit-HRP. Absorbance was measured at 492 $\mathrm{nm}$ after addition of substrate. Percentages of binding were related to DMSO $\left({ }^{*} p<0.01\right)$. 


\section{Discussion}

388 Current COVID-19 pandemic has affected millions of people all around the globe and has been one of the mayor challenges in this century due to a great loss of lives and significant economic losses (1). This highlights an urgent need for developing efficient therapeutics against SARS-CoV-2 since there is no licensed treatment available.

There are several pathogenic viruses, that are known to use the endocytic pathway to enter the cell, the most important being EBOV. Two publications described simultaneously that the endosomal protein called NPC1 or NiemannPick type $\mathrm{C} 1$ is a host receptor for $\operatorname{EBOV}(21,22)$. EBOV entry is mediated by the viral glycoprotein (GP) which is organized in trimeric spikes at the viral surface (20). NPC1 binding requires the processing of viral GP. GP cleavage by endosomal cathepsins unmasks the binding site for NPC1 by removing heavily glycosylated C-terminal residues and the glycan cap to produce the cleaved form of the N-terminal receptor binding subunit GP1 (GPCL). Finally, GPCL-NPC1 binding within endosomes is required to mediate fusion and viral escape into the host cytoplasm $(21,22)$ as a second intracellular receptor $(47)$. Thereby, NPC1 could be used as an important druggable target (22) on viral infection. An example of this is compound U18666A, which blocks intracellular cholesterol efflux mediated by NPC1, along with imipramine severely impacts $\operatorname{EBOV}(22,42,43)$ and other viruses like $\operatorname{HIV}(23)$ and $\operatorname{DENV}(27,28), \operatorname{CHIK}(26)$, ZIKV (29) and other Flavivirus. Knockdown or chemical impairment of NPC1 severely reduced cholesterol supply at the Hepatitis $C$ virus replication sites altering the replication membranous web (25).

SARS-CoV-2 infection starts with the interaction between spike glycoprotein (S) with the ACE2 cellular receptor. It requires activation by the TMPRSS2 at the plasma membrane. TMPRSS2 is located at the vicinity of ACE2 in lipid rafts and elicits plasma membrane fusion (31) that results severely impaired using chemical inhibitors of this protease $(10,11)$. Apart from TMPRSS2, the lysosomal proteases, specifically cathepsin L, are crucial for SARS-CoV-2 entry via endo- 
420 effects along with the cleavage caused by furin at the Golgi (subsequent to S-

421 protein synthesis during viral packaging) on activating SARS-CoV-2 entry and

422 penetration in the cytoplasm.

423 SARS-CoV-2 would traffic the endocytic pathway inside the early and late 424 endosomal vesicles to finally fuse with lysosomes, an essential stage for viral 425 uncoating and fusion (31). According to that, viral infection is abrogated by drugs 426 interfering endosome acidification (11). Also, SARS-CoV-2 pseudovirions 427 infection is inhibited using drugs targeting the late endosomal compartments, like 428 cathepsin L, two-pore channel 2 (TPC2), or PIKfyve. Inhibitors against these 429 proteins dramatically reduce infection, indicating that TPC2, cathepsin L, 430 endosomal maturation, and endosomal acidic luminal $\mathrm{pH}$, are crucial host factors 431 for endocytosed SARS-CoV2 entry $(11,35)$. Thus, late endosomes/lysosomes 432 are proposed as relevant organelles to develop therapeutic targets against 433 infection by SARS-CoV-2 $(8,11,31-34,48)$.

434 A recent study discovered that SARS-CoV-2 non-structural protein 7 (nsp7) 435 strongly interacts with Rab7a, and its depletion causes retention of ACE2 436 receptor inside late endosomes (45). Other reports highlighted the relevance of 437 a variety of proteins involved in cholesterol biosynthesis, including NPC1 infection 438 (32). Also, the cholesterol biosynthesis pathway is downregulated during SARS439 CoV-2 infection and, according to that, drug treatments that regulate this pathway 440 impact the infection (49).

441 Here, we described in this study an interaction between SARS-CoV-2 N protein 442 and NPC1. This interaction unveiled a novel host-based target for antivirals and 443 a potential host factor for SARS-CoV-2 infectivity. As in other viruses, this 444 interaction could possibly regulate and modify cholesterol efflux from late 445 endosomes and alter the lipid composition in cellular membranes in its own 446 benefit (50). Besides, we presented data on how several compounds that block 447 NPC1 function severely impact 229E HCoV infection in a functional assay, which 448 suggests an essential role for NPC1 in HCoV infectivity.

449 Small molecule inhibitors were crucial to determine that NPC1 was essential for 450 EBOV infection (22). Compounds MBX2254, an aminoacetamide sulfonamide, 451 and MBX2270, a triazole thioether were reported to inhibit EBOV infection with 452 high selectivity (41). All those compounds have NPC1 as a target and we found 
453 that those chemicals strongly inhibited HCoV 229E infection. Also, compounds

454 found using the NPC1/EBOV-GP interaction for the screening of a library of

455 compounds, namely sulfides, carbazoles and benzothiazepines (shown in Figure

456 2), were tested for HCoV inhibition. We have shown that compounds that

457 inhibited EBOV-GP/NPC1 binding, namely sulfides SC073 and SC198 together

458 with carbazole SC816, and not others, presented a potent inhibition of 229E-CoV

459 infection. These chemicals have been shown to inhibit EBOV binding to NPC1

460 and the infection of EBOV-GP pseudovirions elsewhere (40). We have shown

461 here that those compounds that were able to inhibit EBOV-GP/NPC1 binding

462 were also capable to inhibit SARS-CoV-2 N protein/NPC1 binding in an ELISA

463 assay.

464 To conclude, according to other authors and recent evidences (31-34, 49), we 465 propose NPC1 as a potential therapeutic target for SARS-CoV-2 to combat 466 COVID-19 pandemic. We show for first-time experimental evidences of the 467 binding of SARS-CoV-2 Nucleoprotein (N) to NPC1. This important finding paves 468 the way to direct medical efforts and therapeutics to NPC1 and to continue 469 studies on cholesterol metabolism in SARS-CoV-2 infection. 


\section{Acknowledgments}

474

475 We are thankful to V. Thiel from the University of Bern, Switzerland for CoV 229E-

476 GFP and T. Pietschman, Twincore, Germany for Huh-7 Lunet C3 cells.

477 BioRender.com was used to created icons in Figures. This research was partially

478 supported through "La Caixa" Banking Foundation (HR18-00469), Instituto de

479 Salud Carlos III (ISCIII-COV20/01007), CSIC (201980E024 and 202020E079),

480 Spanish Ministry of Science and Innovation (RTI2018-097305-R-I00) and the

481 European Commission Horizon 2020 Framework Programme VACDIVA-SFS-

482 12-2019-1-862874.

483

484

485

486

487

488

489

490

491

492

493

494

495

496

497

498

499

500

501

502

503

504

505

506 


\section{Supplementary Figure Legends}

1

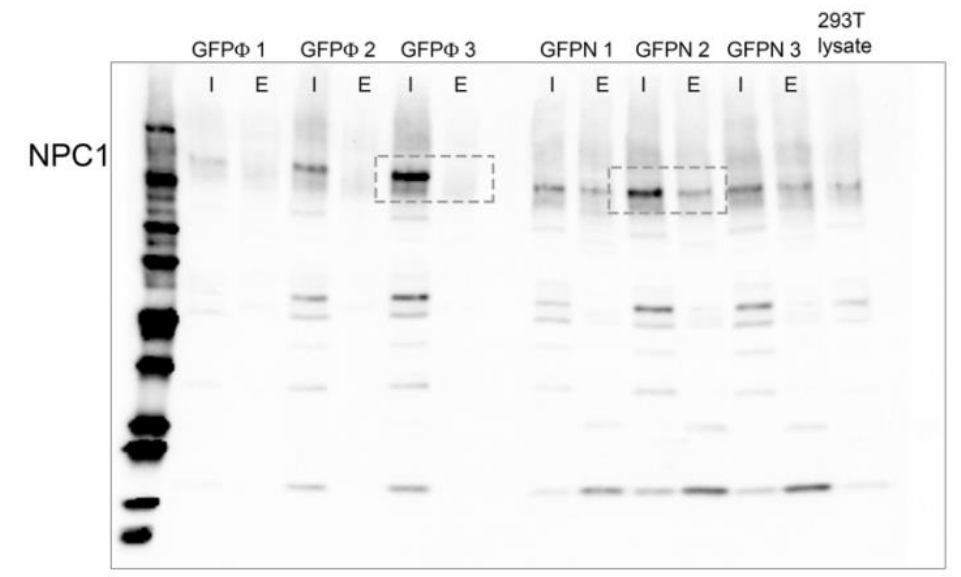

2

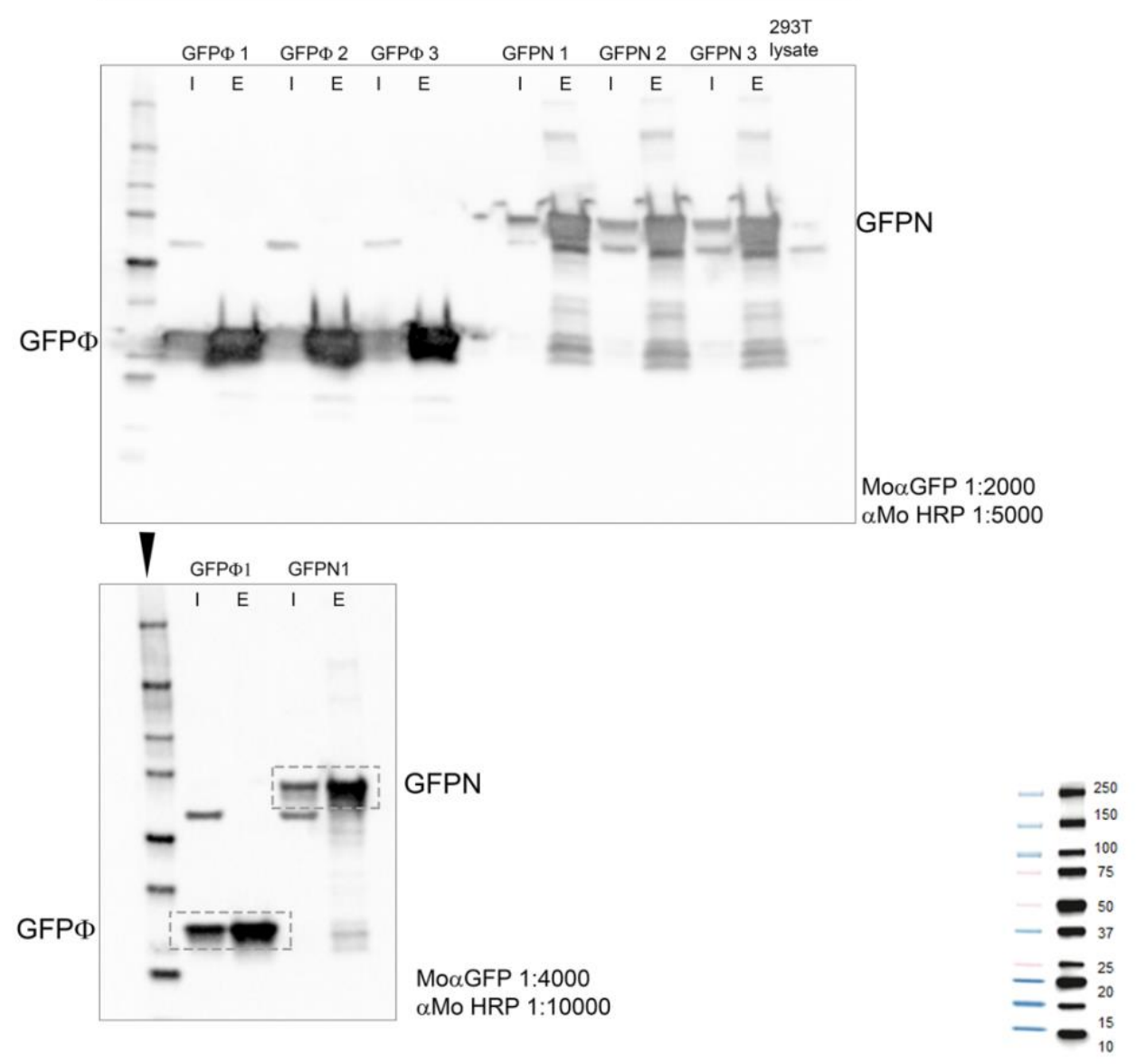

3

Supplementary Figure S1A: Membranes used to compose the figure 1C. Dashed boxes were taken to create the western blot composition showed in the figure. Membrane 1 triplicates revealed with rabbit antiNPC1 antibody, membrane 2 triplicates revealed with mouse anti-GFP antibody and membrane 2 revealed with the same primary and secondary antibodies double diluted. 
bioRxiv preprint doi: https://doi.org/10.1101/2020.12.19.423584; this version posted December 20, 2020. The copyright holder for this preprint (which was not certified by peer review) is the author/funder, who has granted bioRxiv a license to display the preprint in perpetuity. It is made available under aCC-BY-NC-ND 4.0 International license.

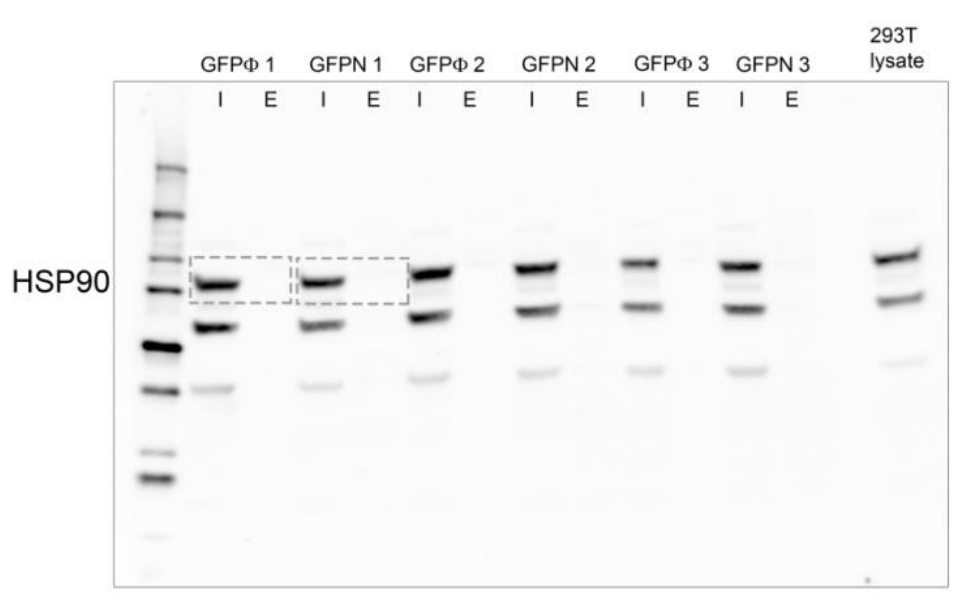

Supplementary Figure S1B (cont): Membrane used to compose the figure 1C. Dashed boxes were taken to create the western blot composition showed in the figure. Original membrane.
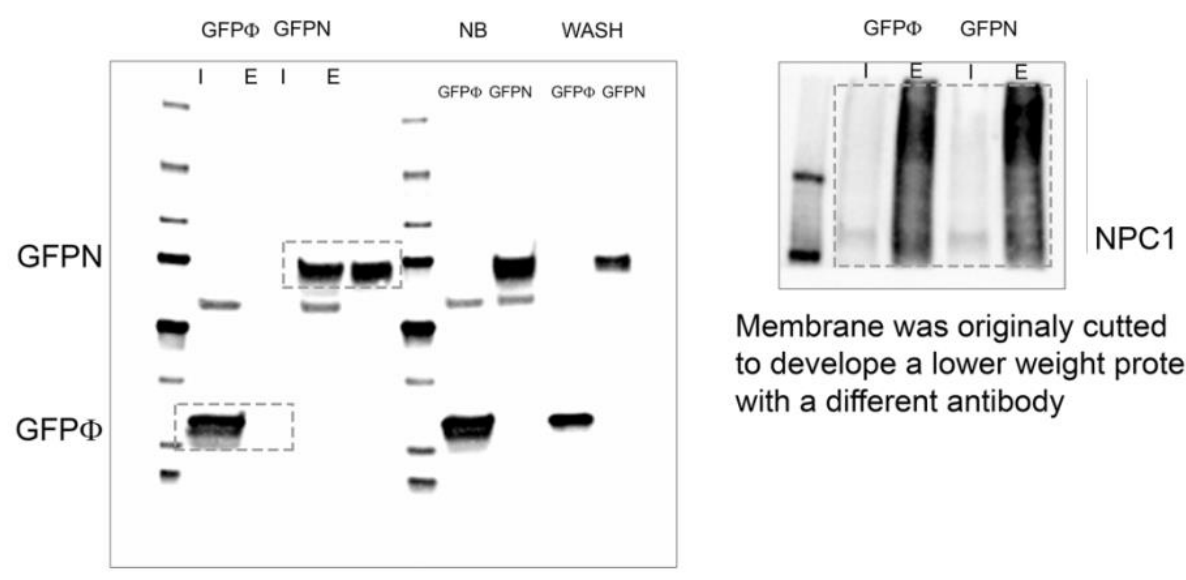

Membrane was originaly cutted to develope a lower weight protein with a different antibody

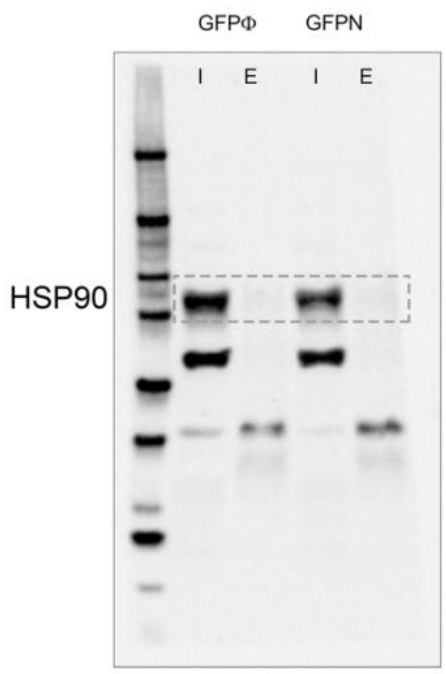

Supplementary Figure S2: Membranes used to compose the figure 1D. Dashed boxes were taken to create the western blot composition showed in the figure. Original membrane 1 developed with Mouse anti-GFP antibody, membrane 2 developed with Rabbit anti-NPC1 antibody and membrane 3 developed with Rat antiHSP90 antibody. 


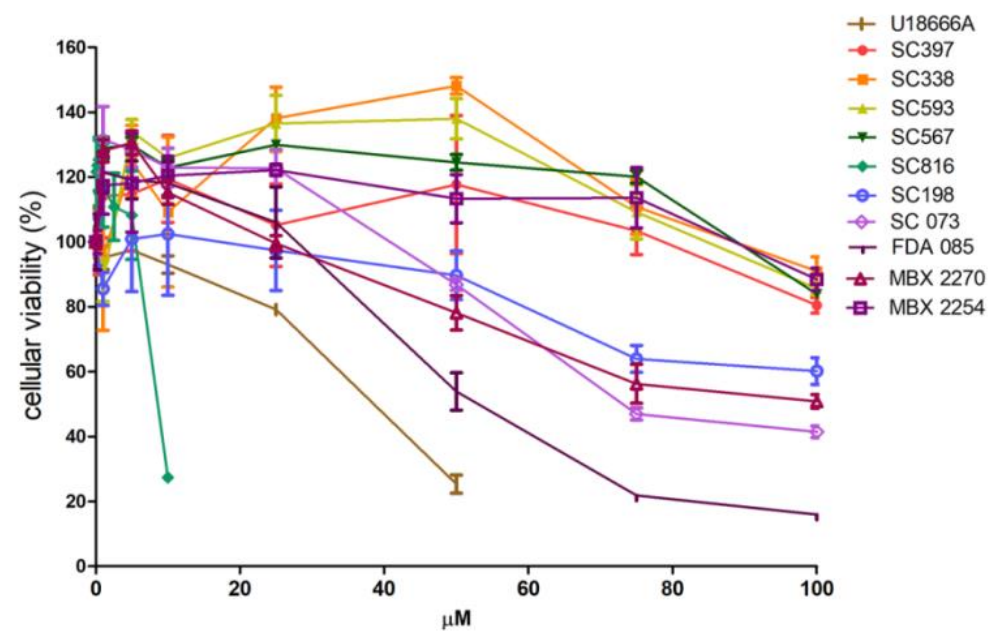

Supplementary Figure S3: Cell viability under chemicals treatment

Cell viability measured after 24 hours incubation of Huh-7 cells with each compound at concentrations ranging from $0-100 \mu \mathrm{M}$ in DMEM. Absorbance was measured at $490 \mathrm{~nm}$ using an ELISA plate reader. $\mathrm{Y}$ axis depicts median and standard deviations of the percentages of absorbance in compound treated cells
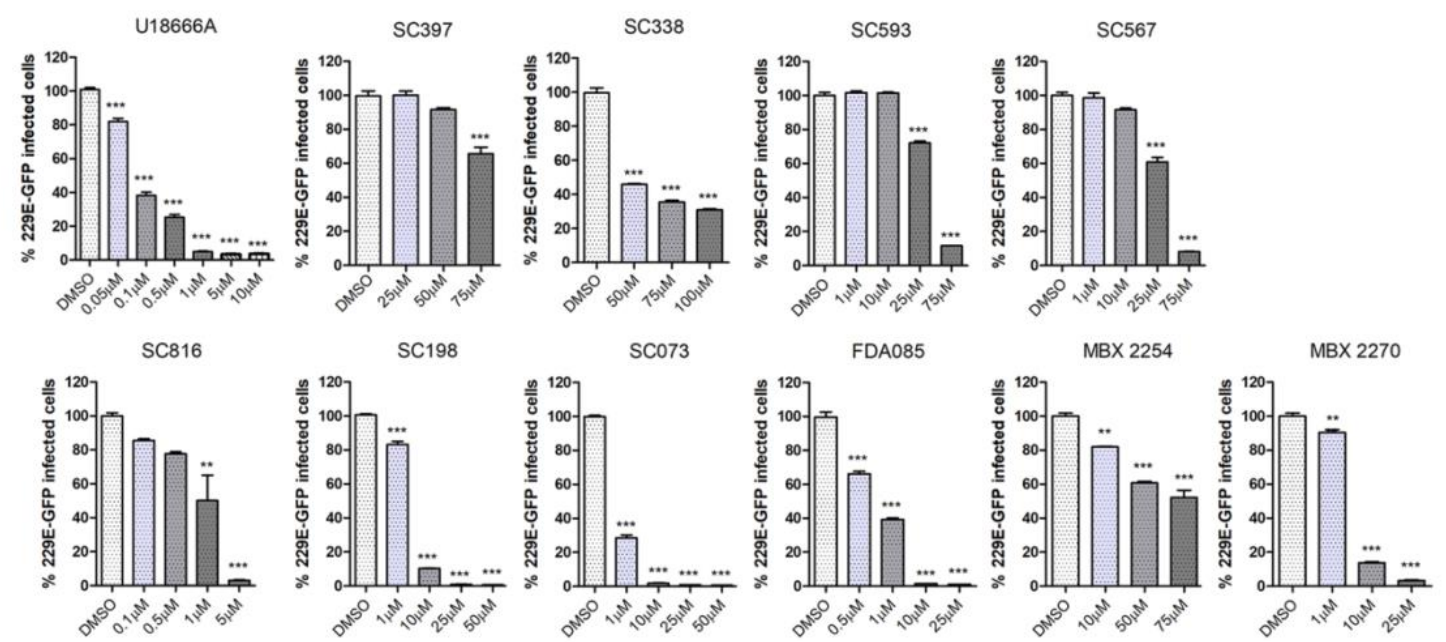

Supplementary Figure S4: Dose-response curves of the compounds used. Dose-response curves with increasing concentrations of compounds at ranges selected depending on working concentrations for each compound. 


\section{Bibliography}

1. $W H O$

WHO

Coronavirus

report,

December

2020. https://www.who.int/emergencies/diseases/novel-coronavirus-2019, (2020).

2. N. Zhu et al., A novel coronavirus from patients with pneumonia in China, 2019.

547 (2020).

3. P. Zhou et al., A pneumonia outbreak associated with a new coronavirus of probable bat origin. 579, 270-273 (2020).

4. J. Lan et al., Structure of the SARS-CoV-2 spike receptor-binding domain bound to the ACE2 receptor. 581, 215-220 (2020).

552

553

554

555

556

557

558

559

5. J. Shang et al., Cell entry mechanisms of SARS-CoV-2. 117, 11727-11734 (2020).

6. J. Shang et al., Structural basis of receptor recognition by SARS-CoV-2. 581, 221-224 (2020).

7. H. Wang et al., SARS coronavirus entry into host cells through a novel clathrinand caveolae-independent endocytic pathway. 18, 290-301 (2008).

8. T. Zhou et al., Cryo-EM Structures of SARS-CoV-2 Spike without and with ACE2 Reveal a pH-Dependent Switch to Mediate Endosomal Positioning of ReceptorBinding Domains. 28, 1-13 (2020).

9. Y. Inoue et al., Clathrin-dependent entry of severe acute respiratory syndrome coronavirus into target cells expressing ACE2 with the cytoplasmic tail deleted. 81, 8722-8729 (2007).

10. M. Hoffmann et al., SARS-CoV-2 cell entry depends on ACE2 and TMPRSS2 and is blocked by a clinically proven protease inhibitor. (2020).

11. X. Ou et al., Characterization of spike glycoprotein of SARS-CoV-2 on virus entry and its immune cross-reactivity with SARS-CoV. 11, 1-12 (2020).

12. B. Coutard et al., The spike glycoprotein of the new coronavirus 2019-nCoV contains a furin-like cleavage site absent in CoV of the same clade. 176, 104742 (2020).

13. A. M. Tharappel, S. K. Samrat, Z. Li, H. J. A. I. D. Li, Targeting Crucial Host Factors of SARS-CoV-2. (2020).

14. S. Klein et al., SARS-CoV-2 structure and replication characterized by in situ cryo-electron tomography. 11, 1-10 (2020).

15. K. Knoops et al., SARS-coronavirus replication is supported by a reticulovesicular network of modified endoplasmic reticulum. 6, e226 (2008).

16. G. Wolff et al., A molecular pore spans the double membrane of the coronavirus replication organelle. 369, 1395-1398 (2020).

17. K. Chandran, N. J. Sullivan, U. Felbor, S. P. Whelan, J. M. J. S. Cunningham, Endosomal proteolysis of the Ebola virus glycoprotein is necessary for infection. 308, 1643-1645 (2005).

18. L. Nathan et al., Calcium ions directly interact with the Ebola virus fusion peptide to promote structure-function changes that enhance infection. 6, 250-260 (2019).

19. P. D. Kwong et al., HIV-1 evades antibody-mediated neutralization through conformational masking of receptor-binding sites. 420, 678-682 (2002).

20. J. M. White, S. E. Delos, M. Brecher, K. J. C. r. i. b. Schornberg, m. biology, Structures and mechanisms of viral membrane fusion proteins: multiple variations on a common theme. 43, 189-219 (2008). 
21. J. E. Carette et al., Ebola virus entry requires the cholesterol transporter Niemann-Pick C1. 477, 340-343 (2011).

22. M. Côté et al., Small molecule inhibitors reveal Niemann-Pick C1 is essential for Ebola virus infection. 477, 344-348 (2011).

23. Y. Tang, I. C. Leao, E. M. Coleman, R. S. Broughton, J. E. J. J. o. v. Hildreth, Deficiency of niemann-pick type C-1 protein impairs release of human immunodeficiency virus type 1 and results in Gag accumulation in late endosomal/lysosomal compartments. 83, 7982-7995 (2009).

24. B. Sainz et al., Identification of the Niemann-Pick C1-like 1 cholesterol absorption receptor as a new hepatitis C virus entry factor. 18, 281-285 (2012).

25. I. K. Stoeck et al., Hepatitis $C$ virus replication depends on endosomal cholesterol homeostasis. 92, (2018).

601

26. S. Wichit et al., Imipramine inhibits chikungunya virus replication in human skin fibroblasts through interference with intracellular cholesterol trafficking. 7, 1-12 (2017).

603

604

27. N. Jupatanakul, S. Sim, G. J. D. Dimopoulos, C. Immunology, Aedes aegypti ML

28. M. K. Poh et al., U18666A, an intra-cellular cholesterol transport inhibitor, inhibits and Niemann-Pick type $C$ family members are agonists of dengue virus infection.

29. C. Sabino et al., Bafilomycin A1 and U18666A efficiently impair ZIKV infection. 11, 524 (2019).

30. J. F. Osuna-Ramos, J. M. Reyes-Ruiz, R. M. J. F. i. c. del Ángel, i. microbiology, The role of host cholesterol during flavivirus infection. 8, 388 (2018).

31. R. A. Ballout, D. Sviridov, M. I. Bukrinsky, A. T. J. T. F. J. Remaley, The lysosome: A potential juncture between SARS-CoV-2 infectivity and Niemann-Pick disease type C, with therapeutic implications. (2020).

32. Z. Daniloski et al., Identification of required host factors for SARS-CoV-2 infection in human cells. (2020).

33. S. Sturley et al., Potential COVID-19 therapeutics from a rare disease: Weaponizing lipid dysregulation to combat viral infectivity. jlr. R120000851 (2020).

34. C. Vial, J. F. Calderón, A. D. J. C. M. M. Klein, NPC1 as a Modulator of Disease Severity and Viral Entry of SARSCoV-2. (2020).

35. I. Galindo et al., Antiviral drugs targeting endosomal membrane proteins inhibit distant animal and human pathogenic viruses. 104990 (2020).

36. L. Cervantes-Barragan et al., Dendritic cell-specific antigen delivery by coronavirus vaccine vectors induces long-lasting protective antiviral and antitumor immunity. 1, (2010).

37. I. García-Dorival et al., Elucidation of the cellular interactome of Ebola virus nucleoprotein and identification of therapeutic targets. 15, 4290-4303 (2016).

38. J. M. Escribano et al., Chrysalises as natural production units for recombinant subunit vaccines. (2020).

39. V. Sebastián-Pérez et al., Medicinal and biological chemistry (MBC) library: an efficient source of new hits. 57, 2143-2151 (2017).

40. F. Lasala et al., Identification of Putative inhibitors of protein-protein Interaction useful to figth against Ebola and other highly pathogenic viruses. In press. Antiviral Research, (2020). 
637 41. A. Basu et al., Novel small molecule entry inhibitors of Ebola virus. 212, S425$638 \quad$ S434 (2015).

639 42. F. Lu et al., Identification of NPC1 as the target of U18666A, an inhibitor of

640 lysosomal cholesterol export and Ebola infection. 4, e12177 (2015).

641

642

43. A. S. Herbert et al., Niemann-pick $\mathrm{C} 1$ is essential for ebolavirus replication and pathogenesis in vivo. 6, (2015).

643

44. I. García-Dorival et al., Elucidation of the Ebola virus VP24 cellular interactome

644

645 and disruption of virus biology through targeted inhibition of host-cell protein function. 13, 5120-5135 (2014).

646

647

45. D. E. Gordon et al., Comparative host-coronavirus protein interaction networks reveal pan-viral disease mechanisms. 370, (2020).

649

46. C. Rodriguez-Lafrasse et al., Abnormal cholesterol metabolism in imipraminetreated fibroblast cultures. Similarities with Niemann-Pick type C disease. 1043,

650 123-128 (1990).

651

47. E. H. Miller et al., Ebola virus entry requires the host-programmed recognition of an intracellular receptor. 31, 1947-1960 (2012).

653

654

655

48. T. Tang, M. Bidon, J. A. Jaimes, G. R. Whittaker, S. J. A. r. Daniel, Coronavirus membrane fusion mechanism offers as a potential target for antiviral development. 104792 (2020).

656

49. D. A. Hoagland et al., Modulating the transcriptional landscape of SARS-CoV-2 as an effective method for developing antiviral compounds. (2020).

658

659

50. L. V. Chernomordik, M. M. J. N. s. Kozlov, m. biology, Mechanics of membrane fusion. 15, 675-683 (2008). 